\title{
Quantitative proteomics analysis of the role of tetraspanin-8 in the drug resistance of gastric cancer
}

\author{
LAN LI*, DAPING YANG* , DEJUN CUI, YU LI, ZHAO NIE, JINGLIN WANG and LI LIANG \\ Department of Gastroenterology, Guizhou Provincial People's Hospital, Guiyang, Guizhou 550003, P.R. China
}

Received August 11, 2017; Accepted December 6, 2017

DOI: 10.3892/ijo.2017.4231

\begin{abstract}
Gastric cancer, due to its high incidence rate, is the second leading cause of cancer-related mortality worldwide. Chemotherapy is an important component of the multimodal treatment for gastric cancer; however, a significant impediment to successful treatment is multidrug resistance (MDR) in patients with gastric cancer. In the present study, the protein profiles of the MDR cell line, SGC7901/DDP, and its parental cell line, SGC7901, were comparatively analyzed through an iTRAQ-based quantitative proteomics technique. The protein tetraspanin-8 (TSPAN8) was found to be highly expressed in the SGC7901/DDP cells. To examine the role of TSPAN8 in the MDR of SGC7901/DDP cells, we increased cell sensitivity to drugs by increasing apoptosis. Additionally, the silencing of TSPAN8 downregulated Wnt pathway activity, $\beta$-catenin expression and $\beta$-catenin transfer to the nucleus. TSPAN8 was found to bind to NOTCH2, facilitating its mediation of the Wnt $/ \beta$-catenin pathway by regulating $\beta$-catenin expression. Overall, the suppression of TSPAN8 expression may prove to be a promising strategy which may aid in the development of novel gastric cancer therapeutic drugs.
\end{abstract}

\section{Introduction}

Globally, gastric cancer is the fourth most common malignancy and the second leading cause of cancer-related mortality, affecting approximately one million individuals each $(1,2)$. Chemotherapy has been applied widely in the treatment of gastric cancer at different stages (3). However, a major issue in the treatment of gastric cancer is the development of resistance to multiple chemotherapeutic agents in tumor cells (4). Multidrug resistance (MDR) in cancer cells is an

Correspondence to: Dr Lan Li, Department of Gastroenterology, Guizhou Provincial People's Hospital, 33 Zhongshan East Road, Guiyang, Guizhou 550003, P.R. China

E-mail: lilanqun@hotmail.com

*Contributed equally

Key words: tetraspanin-8, gastric cancer, drug resistance, isobaric tags for the relative and absolute quantification acquired resistance to multiple drugs, which may be structurally and functionally different (5). Various mechanisms may lead to the development of MDR in cancer cells, including the altered expression of drug influx/efflux transporters, aberrant DNA repair and impairment, the prevention of apoptosis, the mutation of drug targets in targeted therapy, alterations in the cell cycle and checkpoints and an altered tumor microenvironment $(5,6)$. The signaling pathways involved include, in some cancers, Wnt/ $\beta$-catenin, NOTCH and PI3K/AKT, among others, leading to increased resistance to drug treatment with both chemotherapy and targeted therapy (7-10). Interfering with these signaling pathways may be a novel antitumor strategy with which to prevent/inhibit MDR in clinical therapies.

Isobaric tags for the relative and absolute quantification (iTRAQ) analysis is an emerging quantitative proteomics technology that utilizes peptides labeled with isotope-coded covalent tags for the analysis of changes in protein expression in different samples (11). In the present study, the iTRAQ-based proteomic approach was applied to identify differentially expressed proteins in the SGC7901 and SGC7901/DDP cell lines. Among the proteins screened by this approach, tetraspanin-8 (TSPAN8) expression was found to be significantly increased in the SGC7901/DDP cells.

The TSPAN8 gene encodes a cell surface glycoprotein characterized by 4 transmembrane domains and well-conserved cysteine residues in a large extracellular loop, and is expressed in gastric, colon, rectal and pancreatic carcinomas, but not in the majority of normal tissues (12-15). Within the Tetraspanin-enriched microdomain (TEM), TSPAN8 acts as a molecular facilitator (16), being involved in tissue differentiation (17), tumor-cell metastasis (18), and cell motility and cell fusion $(18,19)$. TSPAN8 has been shown to be overexpressed in gastric cancer and to promote cancer cell proliferation, migration and invasion (20). However, the role of TSPAN8 in MDR gastric cancer cells remains unknown. Thus, in the present study, we identified TSPAN8 as a pro-drug resistance protein using iTRAQ-based quantitative proteomics. The silencing of TSPAN8 enhanced the sensitivity of the SGC7901/DDP cells to chemotherapeutic drugs. Additionally, TSPAN8 mediated the activation of the $\mathrm{Wnt} / \beta$-catenin pathway by binding to NOTCH2. These results indicate that TSPAN8 increases the MDR of gastric cancer cells. The inhibition of TSPAN8 may reduce drug resistance and may prove to be a strategy for the clinical treatment of patients with gastric cancer. 


\section{Materials and methods}

Cell culture, transfection and drug treatment. The cell lines used in this study were purchased from the China Center for Type Culture Collection (Wuhan, China). SGC7901/DDP is an MDR gastric cancer cell line in which resistance was induced by cisplatin and it is derived from the human gastric cancer cell line, SGC7901. The cells were cultured in RPMI-1640 medium containing $10 \%$ fetal bovine serum (FBS) (both from Gibco, Grand Island, NY, USA) and $1 \%$ penicillin-streptomycin solution. The biological characteristics of MDR of the SGC7901/DDP cell line were maintained by the addition of $1 \mu \mathrm{g} / \mathrm{ml}$ cisplatin (Sigma-Aldrich, St. Louis, MO, USA) to the complete medium. The cells were incubated in an atmosphere with $5 \%$ carbon dioxide at $37^{\circ} \mathrm{C}$. Three small interfering RNA (siRNA) duplexes targeting human TSPAN8 and a control siRNA were synthesized by GenePharma Co., Ltd. (Shanghai, China). The sequences of the siRNA-TSPAN8 were as follows: Sequence 1 forward, 5'-GUAUCUUGAUCCUAGCAUUd TdT-3' and reverse, 5'-AAUGCUAGGAUCAAGAUACdTdT-3'; sequence 2 forward, 5'-GUCUGAUCGCAUUGUGAAUd TdT-3' and reverse, 5'-AUUCACAAUGCGAUCAGACdTdT-3'; sequence 3 forward, 5'-GAGUUUAAAUGCUGCGGUUd TdT-3' and reverse, 5'-AACCGCAGCAUUUAAACUCdTdT-3'; and siRNA-NC forward, 5'-UUCUUCGAAGGUGUCACG UTT-3' and reverse, 5'-ACGUGACACGUUCGGAGAATT-3'. The SGC7901/DDP cells were transfected with the siRNA using siRNA-Mate (GenePharma Co., Ltd.) following the manufacturer's instructions. The inhibitors of the Wnt pathway (CCT036477 and XAV939) and the inhibitor of NOTCH2 (DAPT) were purchased from Santa Cruz Biotechnology, (Santa Cruz, CA, USA). All these drugs were suspended in dimethyl sulfoxide (DMSO; Sangon Biotech, Shanghai, China) at a stock concentration according to the manufacturer's instructions and stored at $-80^{\circ} \mathrm{C}$. Following siRNA transfection for $48 \mathrm{~h}$, the cells were exposed to the inhibitors, which were diluted into the culture medium $(10 \mu \mathrm{M}$ CCT036447, $10 \mu \mathrm{M}$ XAV939, $20 \mu \mathrm{M}$ DAPT or DMSO alone as a control) for $48 \mathrm{~h}$, respectively.

Determination of half maximal inhibitory concentration $\left(I C_{50}\right)$. The cytotoxic effects of the cisplatin, 5-fluorouracil and adriamycin (both from Sangon Biotech) on the SGC7901 and SGC7901/DDP cells were measured by cell counting kit-8 (CCK-8) assay (21). The cells were counted using the Neubauer cell-counting chamber (BRAND GMBH + CO KG, Wertheim, Germany) following the manufacturer's instructions. The cells were then seeded in 96-wells at a density of $5 \times 10^{3}$ cells/well, and cultured in an incubator at $37^{\circ} \mathrm{C}$ for $24 \mathrm{~h}$ before being treated with the chemotherapeutic drugs. Cisplatin, 5-fluorouracil (5-Fu) and adriamycin in graded concentrations were added to the cells. Following treatment for $48 \mathrm{~h}$, the medium was replaced with fresh medium containing 10\% CCK-8 reagent (Dojindo, Kumamoto, Japan), and the cells were incubated for an additional 1-4 h. The optical density was then measured by Thermo Scientific Varioskan Flash spectral scanning multimode reader (Thermo Fisher Scientific, Waltham, MA, USA) at $450 \mathrm{~nm}$. The $\mathrm{IC}_{50}$ values obtained following treatment of the SGC7901 and SGC7901/DDP cells with each drug were analyzed using IBM
SPSS Statistics v21 software (SPSS Inc., Chicago, IL, USA) via probit analysis (22).

Reverse transcription-quantitative PCR (RT-qPCR). Total RNA was extracted using a High Purity Total RNA Rapid Extraction kit (RP1201; BioTeke, Beijing, China) according to the manufacturer's instructions. cDNA was synthesized using a iSCRIPT cDNA Synthesis kit (GeneCopoeia Co., Ltd., Guangzhou, China). The primers used for the amplification of TSPAN8, $\beta$-catenin, NOTCH 2 and glyceraldehyde 3-phosphate dehydrogenase (GAPDH) were synthesized by GeneCopoeia Co., Ltd. GAPDH was used as an internal standard, and the relative expression of each gene was normalized to GAPDH. The real-time PCR kit was purchased from GeneCopoeia Co., Ltd. PCR cycling conditions were as follows: $95^{\circ} \mathrm{C}$ for $10 \mathrm{~min}$, followed by 40 cycles of $95^{\circ} \mathrm{C}$ for $10 \mathrm{sec}, 60^{\circ} \mathrm{C}$ for $20 \mathrm{sec}$ and $72^{\circ} \mathrm{C}$ for $10 \mathrm{sec}$. The relative quantification of gene expression was analyzed using the $2^{-\Delta \Delta C t}$ method (23). Each sample was analyzed in triplicate.

Western blot analysis. Protein was extracted from the cells using RIPA lysis buffer (Beyotime, Shanghai, China) and the concentration was determined using the 2D Quantification kit (Amersham Biosciences, Little Chalfont, UK). The protein samples were separated on a $10 \%$ polyacrylamide gel, and electrotransferred onto polyvinylidene fluoride membranes (Millipore Corp., Billerica, MA, USA). The membranes were then blocked with $5 \%$ non-fat dried milk for $1 \mathrm{~h}$ at room temperature. This was followed by the addition of the primary antibodies: anti-TSPAN8 antibody (ab70007), anti- $\beta$-catenin antibody (ab16051) (both from Abcam, Cambridge, MA, USA), anti-cellular retinoic acid-binding protein 2 (CRABP2) antibody (10225-1-AP), anti-voltage-dependent anion-selective channel protein 2 (VDAC2) antibody (11663-1-AP), anti-Bcl-2 antibody (12789-1-AP) (all from Proteintech, Wuhan, China), anti-heat shock protein 90 (HSP90) antibody (bs-0889R), anti-erythrocyte membrane protein band 4.1 (EPB41) antibody (bs-13080R), antitumor protein D54 (TPD54) antibody (bs-6743R), anti-mucin 13 (MUC13) antibody (bs-10074R), anti-GAPDH antibody (bs-10900R), anti-caspase-3 antibody (bs-0081R) and antiBax antibody (bs-0127R) (all from Bioss, Beijing, China) and overnight incubation at $4^{\circ} \mathrm{C}$. All primer antibodies were diluted $(1: 1,000)$ by Tris-buffered saline containing $0.1 \%$ Tween-20 (TBS-T) After washing 3 times with TBS-T, the membranes were incubated with a horseradish peroxidase-conjugated goat antirabbit IgG as a secondary antibody (1:5,000, ab6721; Abcam) for $2 \mathrm{~h}$ at room temperature. After washing 3 times with TBS-T buffer, the membranes were visualized with an ECL detection system (KeyGen Biotech Inc., Nanjing, China). All western blot analyses were repeated at least 3 times.

Luciferase reporter assay. The cells were seeded in a 6-well plate and transfected with siRNA according to the protocol of siRNA-Mate (GenePharma Co., Ltd.). TOP-flash reporter plasmid was purchased from Shanghai Qcbio Science and Technologies Co., Ltd. (Shanghai, China) and was transfected into the cells by Endofectin ${ }^{\mathrm{TM}}$-Plus (GeneCopoeia) according to the manufacturer's instructions $48 \mathrm{~h}$ after siRNA transfection. The reporter gene assay was performed $48 \mathrm{~h}$ post-plasmid-transfection using the Dual Luciferase Assay 
System (Promega, Madison, WI, USA). Firefly luciferase activity was normalized for transfection efficiency using the corresponding Renilla luciferase activity. All experiments were performed at least in triplicate.

Immunoprecipitation. The plasmids (HA-TSPAN8, Flag-TSPAN8, HA-NOTCH2 and Flag-NOTCH2) used for exogenous co-immunoprecipitation were synthesized by GeneCopoeia Co., Ltd. The cells were lysed by sonication and centrifugation at $4^{\circ} \mathrm{C}, 16,000 \mathrm{x} \mathrm{g}, 10 \mathrm{~min}$ (TDZ4-WS centrifuge; Thermo Fisher Scientific) in IP lysis buffer (Beyotime, Beijing, China) supplemented with phosphatase/protease inhibitor cocktail and $1 \mathrm{mM}$ PMSF. The supernatant was transferred to a separate microfuge tube, pre-cleared with protein A/G agarose beads (Yanji Biotechnology, Shanghai, China) and centrifuged at $4^{\circ} \mathrm{C}, 16,000 \times \mathrm{g}, 5 \mathrm{~min}$ (TDZ4-WS centrifuge; Thermo Fisher Scientific) to pellet the beads and remove protein impurities. The supernatant was collected and incubated with rabbit IgG (bs-0295P; Bioss) overnight at $4^{\circ} \mathrm{C}$. The beads were collected by centrifugation at $4^{\circ} \mathrm{C}, 16,000 \mathrm{x} \mathrm{g}$, 10 min (TDZ4-WS centrifuge; Thermo Fisher Scientific), washed 3 times with IP lysis buffer and resuspended with 2X sodium dodecyl sulfate (SDS) loading buffer. Bound protein was eluted off the beads by boiling and examined by western blot analysis as described above.

Immunofluorescence. The cells were incubated with $4.0 \%$ paraformaldehyde for $15 \mathrm{~min}$ at room temperature. The cells were then washed 3 times with phosphate-buffered saline (PBS). To increase permeability, $0.1 \%$ Triton X-100 was added to the cells for $10 \mathrm{~min}$. The cells were then washed again thrice with PBS. The anti- $\beta$-catenin antibody (ab16051; 1:100 diluted by PBS; Abcam) was added to the wells followed by incubation overnight at $4^{\circ} \mathrm{C}$. The cells were then washed and incubated in Alexa Fluor-conjugated secondary antibody (1:100 diluted with Bioss antifade mounting medium; Bioss). DAPI (Invitrogen, Carlsbad, CA, USA) was used to dye the nuclei. The cells were incubated with DAPI for $20 \mathrm{~min}$ at room temperature. After being washed 3 times with PBS, the cells were imaged under a microscope (Ci-L; Nikon, Tokyo, Japan).

Protein extraction and $i T R A Q$ labeling. Total protein extracts were prepared in lysis buffer [7 $\mathrm{M}$ urea, $1 \mathrm{mg} / \mathrm{ml}$ DNase I, $1 \mathrm{mM} \mathrm{Na} \mathrm{VO}_{4}$ (all from Sangon Biotech), and $1 \mathrm{mM}$ PMSF (Bioss, Beijing, China)] using the Sample Grinding kit from Amersham Biosciences. Following being centrifuged at $17,000 \mathrm{x} \mathrm{g}$ for $15 \mathrm{~min}$ at $4^{\circ} \mathrm{C}$, the supernatant was collected and the protein concentrations were quantified with a 2-D Quantification kit (Amersham Biosciences).

From each sample, $100 \mu \mathrm{g}$ of protein was precipitated, denatured, cysteine-blocked and digested with sequencing-grade modified trypsin, according to the manufacturer's instructions (iTRAQ Reagent 8 Plex Multi-plex; Applied Biosystems, Foster City, CA, USA). The samples were then labeled with the iTRAQ tags (SGC7901, 113, 115 tags; SGC7901/DDP 114, 116 tags; Applied Biosystems). The labeled samples were pooled prior to further analysis.

Fractionation of peptides. The iTRAQ-labeled samples were solubilized in $300 \mu \mathrm{l}$ of $1 \%$ Pharmalyte (Amersham
Biosciences) and $8 \mathrm{M}$ urea solution. The samples were rehydrated on IPG gel strips (pH 3.0-10.0; Amersham Biosciences) at $30 \mathrm{~V}$ for $14 \mathrm{~h}$. The peptides were subsequently focused successively at $500 \mathrm{~V}$ for $1 \mathrm{~h}, 1,000 \mathrm{~V}$ for $1 \mathrm{~h}, 3,000 \mathrm{~V}$ for $1 \mathrm{~h}$ and $8,000 \mathrm{~V}$ for $8.5 \mathrm{~h}$. Following electrofocusing, the peptides were extracted from the gel using a solution containing $0.1 \%$ formic acid and $2 \%$ acetonitrile for $1 \mathrm{~h}$. The fractions were then purified and concentrated on a C18 Discovery DSC-18 SPE column (Sigma-Aldrich), lyophilized and maintained at $-20^{\circ} \mathrm{C}$.

Mass spectrometry. The samples were analyzed using a QStar Elite hybrid mass spectrometer (Applied Biosystems) coupled with a liquid chromatography system (Amersham Biosciences, Little Chalfont, UK).

The mass spectrometer was set to perform informationdependent acquisition (IDA) in the positive ion mode at a mass range of $300-1800 \mathrm{~m} / \mathrm{z}$. Peptides with +2 to +4 charge states were selected for tandem mass spectrometry, and the time of summation of MS/MS events was set to $3 \mathrm{sec}$. We selected the two most abundantly charged peptides above a 20 -count threshold for MS/MS and dynamic exclusion was set to $30 \mathrm{sec}$ with a $50 \mathrm{mDa}$ mass tolerance. Data were processed using ProteinPilot version 2.0 software (Applied Biosystems) and searched against the UnitProt (http://www.uniprot.org/) human protein database (v3.77). Protein identification was based on selection thresholds of ProtScore $>1.3$ or ProtScore $<0.77$, and false discovery rate P-values $<0.05$.

Bioinformaticsanalysis. The resultsobtainedbyiTRAQ-labeled proteomics were analyzed using by protein analysis using the evolutionary relationships (PANTHER) classification system (www.pantherdb.org) following the instructions available online (24). STRING 10.5 (http://string.embl.de/) was used to predict the interaction between proteins following the instruction online (25).

Statistical analysis. The in vitro experiments were repeated at least 3 times. Data are presented as the means \pm standard deviation (SD). Significance between groups from in vitro experiments was determined using the Student's t-test or Dunnett's T3 test. A value of $\mathrm{P}<0.05$ was considered to indicate a statistically significant difference.

\section{Results}

iTRAQ-coupled 2D LC-MS/MS analysis of differentially expressed proteins. To identify potential proteins associated with resistance to cisplatin, iTRAQ-based quantification was performed on proteins isolated from cisplatin-sensitive gastric cancer cells (SGC7901) and from DDP-resistant gastric cancer cells (SGC7901/DDP). The specimens were iTRAQ-labeled in duplicate in order to verify the results. Protein samples were labeled as follows: SGC7901, tags 113 and 114; SGC7901/DDP, tags 115 and 116. The relative abundance of protein from the SGC7901/DDP cells with respect to proteins from SGC7901 cells was calculated as the iTRAQ ratios 115:113 and 116:114. These fractions were analyzed by LC/MS/MS. The workflow of the iTRAQ proteomics approach is presented in Fig. 1. ProteinPilot 2.0 software was 


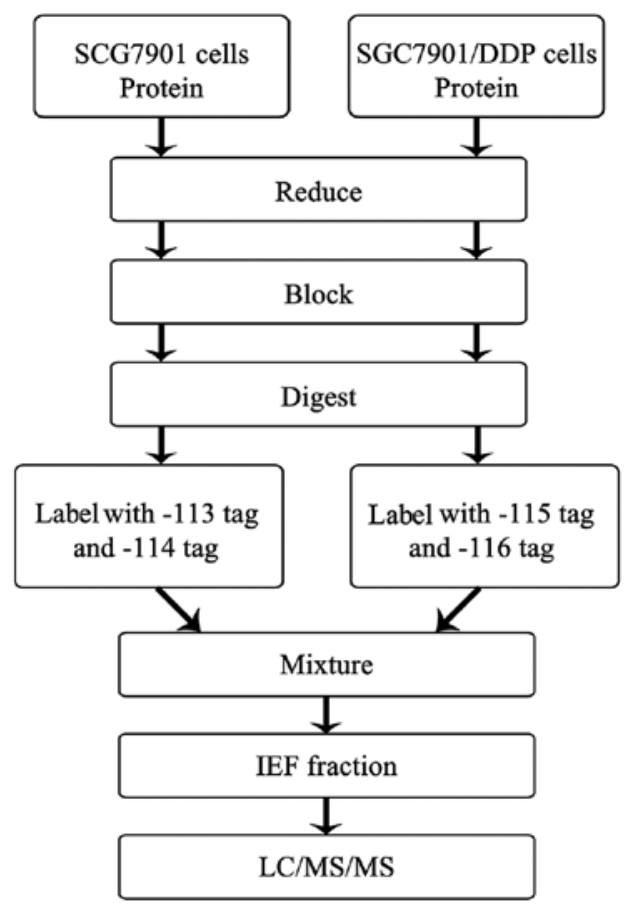

Figure 1. Flowchart of the isobaric tags for the relative and absolute quantification (iTRAQ)-based MS proteomics approach used in this study. used for protein quantification and identification. Considering the technical variations of the method and statistical analysis in the relative quantification analysis, and in order to reduce false-positives and increase accuracy, a 1.3-fold cut-off for all iTRAQ ratios was used $(26,27)$. Therefore, proteins with iTRAQ ratios $<0.77$ - or $>1.3$-fold cut-off $(\mathrm{P}<0.05)$ were considered to be downregulated or upregulated, respectively. A total of 1,324 differentially expressed proteins were identified, regardless of whether or not there was a significant $\mathrm{P}$-value in the iTRAQ ratios. Of these, 112 proteins were differentially expressed in the SCG7901/ddp cells compared to the SGC7901 cells (64 upregulated and 48 downregulated proteins). The top 30 downregulated and upregulated proteins are shown in Table I.

Cellular and molecular functional characteristics of the proteins. The 112 proteins, which were potentially differentially expressed between the SGC7901/DDP cells and SGC7901 cells, were classified into 5 functional categories using the Protein Analysis through Evolutionary Relationships (PANTHER) classification system (Fig. 2). The molecular function categories were binding $(23.5 \%)$, receptor activity $(2.9 \%)$, structural molecule activity (14.7\%), catalytic activity (44.1\%) and transporter activity (14.7\%) (Fig. 2).

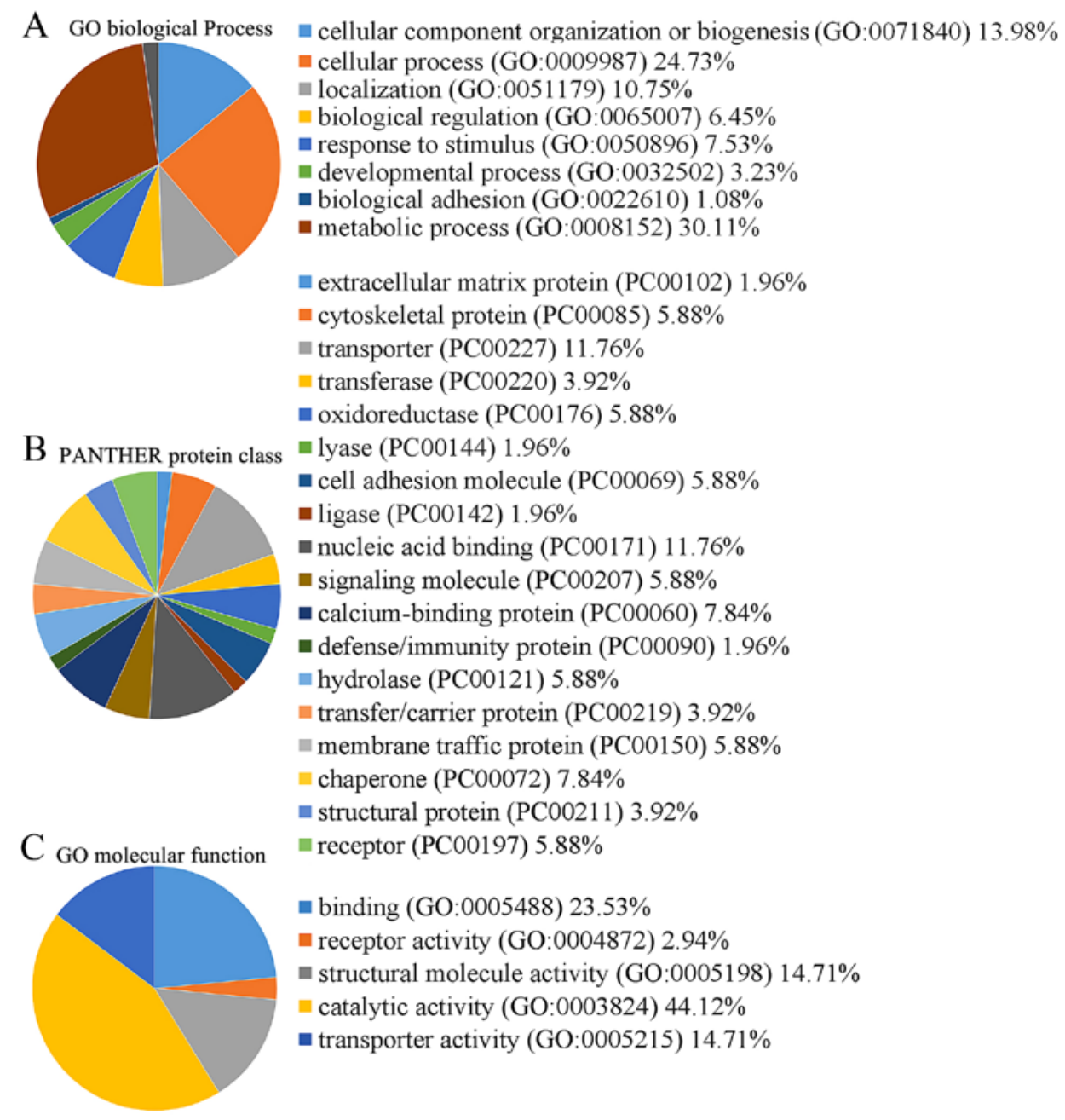

Figure 2. Classification of proteins identified through proteomics into their (A) GO biological process, (B) Protein Analysis through Evolutionary Relationships (PANTHER) protein class, and (C) GO molecular processes. This was carried out using the PANTHER Classification system (www.pantherdb.org/). 
A

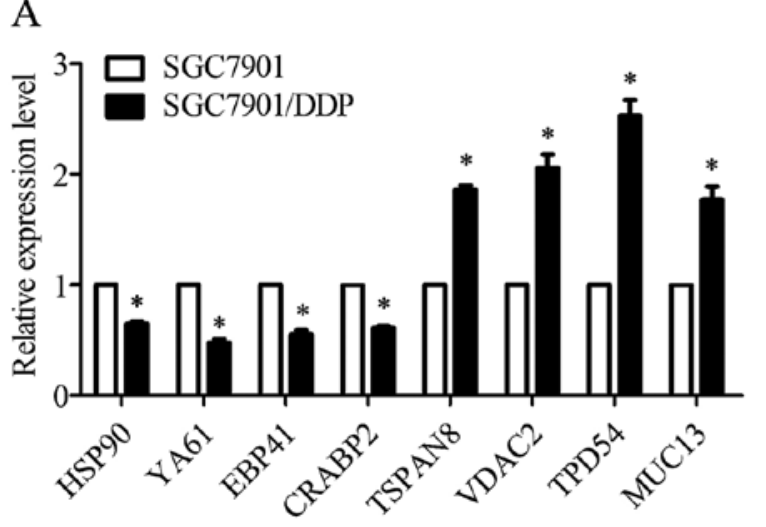

B

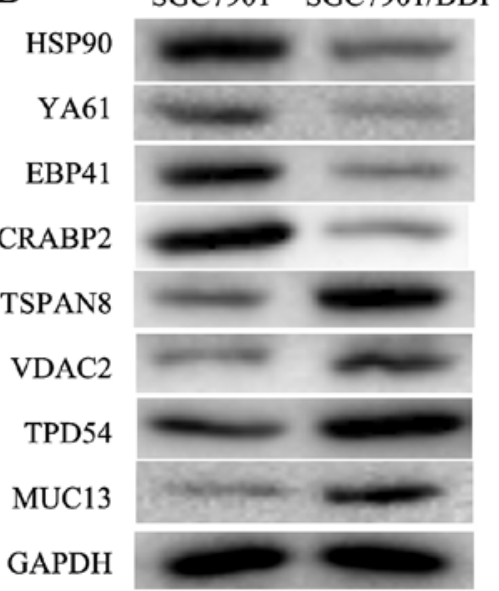

Figure 3. Validation of heat shock protein 90 (HSP90), drug-sensitive protein 1 (YA61), erythrocyte membrane protein band 4.1 (EPB41), cellular retinoic acid-binding protein 2 (CRABP2), tetraspanin-8 (TSPAN8), voltage-dependent anion-selective channel protein 2 (VDAC2), tumor protein D54 (TPD54) and mucin 13 (MUC13) expression in the SGC7901 and SGC7901/DDP cells. (A) RT-qPCR was used to detect the relative mRNA expression levels of HSP90, YA61, EPB41, CRABP2, TSPAN8, VDAC2, TPD54 and MUC13, as normalized to glyceraldehyde 3-phosphate dehydrogenase (GAPDH) (P<0.05). (B) Representative western blot analyses for HSP90, YA61, EPB41, CRABP2, TSPAN8, VDAC2, TPD54 and MUC13 expression in cells. GAPDH was used as the normalization standard. Data are the means $\pm \mathrm{SD} ;{ }^{*} \mathrm{P}<0.05$ vs. negative control (NC).

A

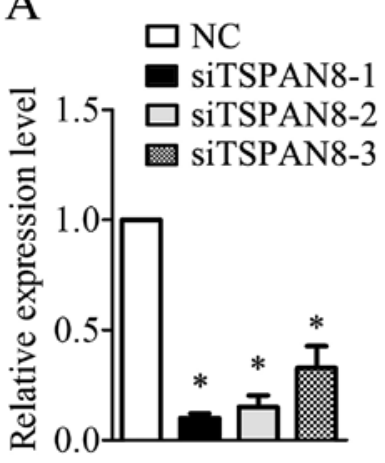

B
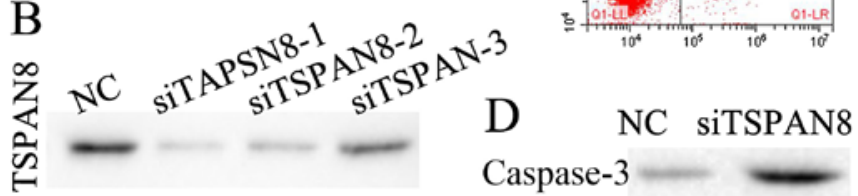

Figure 4. Validation of the silencing of tetraspanin-8 (TSPAN8) using siRNA. (A) The mRNA expression of TSPAN8 was determined by RT-qPCR. TSPAN8 was effectively silenced at the mRNA level by siRNA sequences. (B) The protein expression of TSPAN8 was determined by western blot analysis. TSPAN8 was effectively silenced at the protein level by siRNA sequences. (C) Cell apoptosis was examined by Annexin V FITC-A flow cytometry. Apoptosis was increased in the TSPAN8-silenced SGC7901/DDP cells. (D) The protein expression of caspase-3, Bax and Bcl-2 was examined by western blot analysis. In TSPAN8-silenced SGC7901/DDP cells, the expression of caspase- 3 and Bax was increased, while that of Bcl-2 was decreased. Data are the means $\pm \mathrm{SD} ;{ }^{*} \mathrm{P}<0.05$ vs. negative control (NC).

Validation of differentially expressed proteins. The differentially expressed proteins identified by ITRAQ were validated by RT-qPCR and western blot analysis. The proteins selected for validation were the ones most significantly dysregulated according to protein classification or the ones closely related to multidrug resistance. TSPAN8 has been reported to promote the proliferation and metastasis of SGC7901 cells. The results from iTRAQ-coupled 2D LC-MS/MS revealed that TSPAN8 was potentially related to drug resistance in the SGC7901/DDP cells. Thus, it was selected as the object of the following analysis. The mRNA levels of HSP90, drug-sensitive protein 1 (YA61), EPB41 and CRABP2 were decreased in the SGC7901/DDP cells when compared with those in the SGC7901 cells, whereas the mRNA levels of TSPAN8, VDAC2, TPD54 and MUC13 were increased (Fig. 3A). The results of western blot analysis revealed that the protein expression levels of HSP90, YA61, EPB41 and CRABP2 were downregulated in the SGC7901/DDP cells when compared to those in the SGC7901 cells, whereas the levels of TSPAN8, VDAC2, TPD54 and MUC13 were upregulated (Fig. 3B). These results were consistent with the trend observed in iTRAQ analysis.

Silencing of TSPAN8 in SGC7901/DDP cells reduces MDR. TSPAN8 has been reported to be an oncoprotein in gastric cancer, enhancing gastric cancer cell proliferation and metastasis (20). However, the role of TSPAN8 in gastric cancer cell drug resistance remains unclear. In the present study, TSPAN8 was knocked down by siRNA. RT-qPCR and western blot analysis confirmed the efficacy of the silencing of TSPAN8. As shown in Fig. 4A, the relative mRNA level of TSPAN8 was significantly decreased following transfection with siRNA against TSPAN8. The results of western blot analysis revealed that TSPAN8 protein expression in specific siRNA-transfected SGC7901/DDP cells was effectively suppressed (Fig. 4B). Of the 3 siRNA sequences, sequence 1 was found to be the most suitable for our purposes (Fig. 4A and B), and was thus used in all subsequent experiments.

MDR is the main cause of chemotherapy failure in gastric cancer treatment. Thus, in this study, to assess the association between TSPAN8 and MDR, the siRNA-transfected 

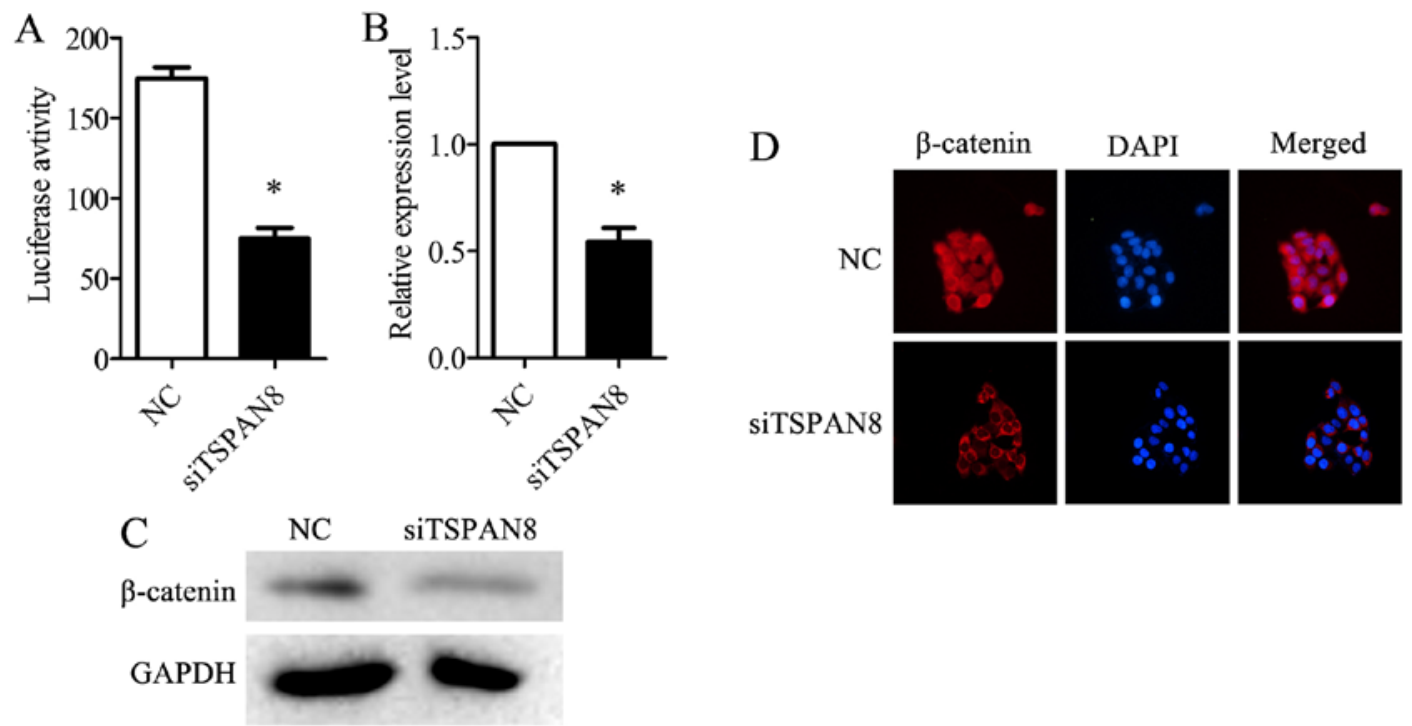

Figure 5. Tetraspanin-8 (TSPAN8) silencing decreases Wnt/ק-catenin activity. (A) Wnt pathway activity was examined by luciferase reporter assay. Wnt activity was decreased in the TSPAN8-silenced SGC7901/DDP cells. The expression of $\beta$-catenin was examined at (B) the mRNA level using RT-qPCR and at (C) the protein level using western blot analysis. (D) The expression of $\beta$-catenin was decreased at both the mRNA and protein level in TSPAN8-silenced SGC7901/ DDP cells. The transfer of $\beta$-catenin into the nucleus was determined using immunofluorescence. The red fluorescence represents $\beta$-catenin, while the blue fluorescence represents the nucleus of SGC7901/DDP cells. The pink color in the merged photos represents $\beta$-catenin in the nucleus. When TSPAN8 was silenced, the $\beta$-catenin staining in the nucleus was less than that in the negative control (NC) group. Data are the means $\pm \mathrm{SD}$; ${ }^{\text {P }} \mathrm{P}<0.05$ vs. negative control NC.

SCG7901/DDP cells were treated with cisplatin, 5-Fu and adriamycin (the most commonly used drugs in clinical practice for the chemotherapeutic treatment of gastric cancer), for 2 days and the $\mathrm{IC}_{50}$ values were determined. The $\mathrm{IC}_{50}$ values of cisplatin, 5-Fu and adriamycin were significantly decreased in the TSPAN8-silenced SGC7901/DDP cells compared with the negative controls (Table II). This result suggested that the silencing of TSPAN8 reduced the resistance of the SGC7901/DDP cells to the aforementioned drugs, which, in turn, indicated that TSPAN8 may contribute to the MDR of this cell line. In the following experiments, only cisplatin was used to maintain the drug resistance of the SGC7901/DDP cells.

Furthermore, compared with the negative control SGC7901/DDP cells, apoptosis was increased in the TSPAN8-silenced cells (Fig. 4C). Moreover, the levels of apoptosis-related proteins (caspase-3, Bax and Bcl-2) were examined by western blot analysis. The results (Fig. 4D) revealed that the levels of caspase- 3 and Bax were upregulated, while those of $\mathrm{Bcl}-2$, an anti-apoptotic protein, were downregulated in the TSPAN8-silenced SGC7901/DDP cells. These results indicated that the silencing of TSPAN8 promoted SGC7901/DDP cell apoptosis.

Silencing of TSPAN8 sensitizes SGC7901/DDP cells to chemotherapy by mediating Wnt/ $\beta$-catenin. Thus far, our findings suggested that TSPAN8 plays a critical role in the drug resistance of SGC7901/DDP cells. It is believed that metastasis is the persistence of cancer stem cells (CSCs), which are highly resistant to chemotherapy (28). The Wnt/ $\beta$-catenin signaling pathway has been reported to increase gastric cancer cell migration and invasion (29). Therefore, in this study, we investigated whether TSPAN8-mediated gastric cancer cell drug resistance is also related to the $\mathrm{Wnt} / \beta$-catenin pathway. The Wnt/ $\beta$-catenin pathway activity was detected using a TOP-flash luciferase reporter. The silencing of TSPAN8 in the SGC7901/DDP cells significantly decreased TOP-flash luciferase activity (Fig. 5A). The TSPAN8-silenced cells displayed a decreased expression of $\beta$-catenin at both the mRNA (Fig. 5B) and protein level (Fig. 5C), compared to negative control (NC)-infected SGC7901/DDP cells. Additionally, the accumulation of $\beta$-catenin in the nucleus was impaired in the TSPAN8-silenced SGC7901/DDP cells (Fig. 5D). The cells were treated with CCT036477 (CCT) and XAV939 (inhibitors of the Wnt- $\beta$-catenin pathway) (30). The reduced $\mathrm{IC}_{50}$ value caused by TSPAN8 silencing was partially reversed when the Wnt- $\beta$-catenin pathway inhibitors were added (Table III). These data indicated that TSPAN8 enhanced the resistance of the SGC7901/DDP cells to chemotherapy through the activation of the Wnt/ $\beta$-catenin pathway and by increasing $\beta$-catenin expression and accumulation in the nucleus. However, compared to the NC group, the inhibitors of the Wnt pathway still decreased the $\mathrm{IC}_{50}$ values (Table III).

TSPAN8 mediated Wnt/ $\beta$-catenin through binding to NOTCH2. To identify which protein or proteins interact with TSPAN8, we utilized STRING 10.5. NOTCH2 was predicted to interact with TSPAN8. Co-immunoprecipitation was used to validate the association between TSPAN8 and NOTCH2. Endogenous co-immunoprecipitation assays revealed that TSPAN8 interacted with NOTCH2 in the SGC7901/DDP cells (Fig. 6A). Consistent with this result, the exogenous interaction between TSPAN8 and NOTCH2 was also observed in the SGC7901/DDP cells that were co-transfected with HA-TSPAN8 and Flag-NOTCH2 (Fig. 6B). These findings revealed that TSPAN8 acts in combination with NOTCH2 in gastric cancer cells. Furthermore, we found that the impairment of $\beta$-catenin expression was partially compensated when DAPT (30), a NOTCH2 inhibitor, was used in the TSPAN8-silenced SGC7901/DDP cells (Fig. 6C and D). The results data indicated that TSPAN8 mediated the activation of the Wnt/ $\beta$-catenin pathway by binding to $\mathrm{NOTCH} 2$. 


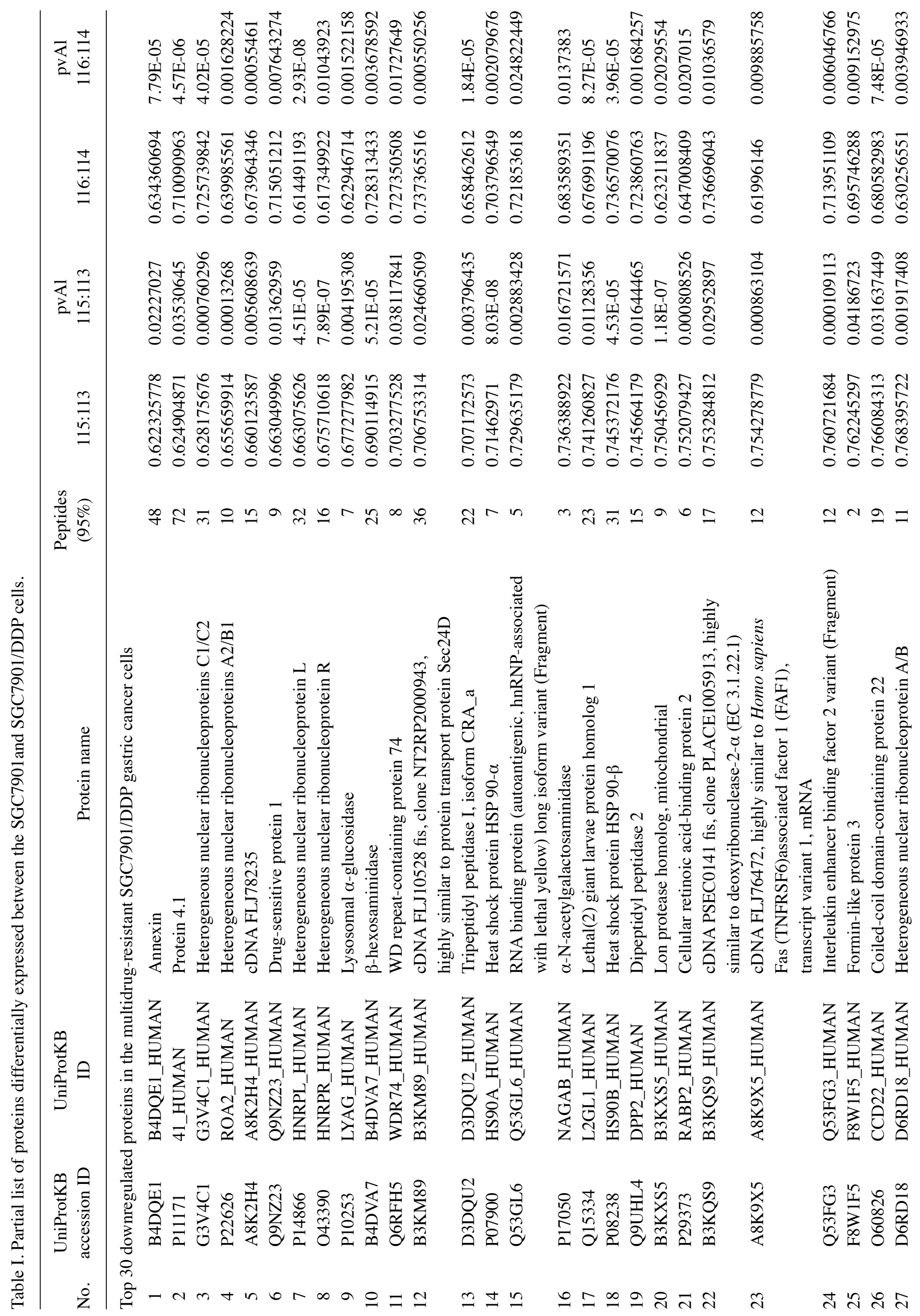




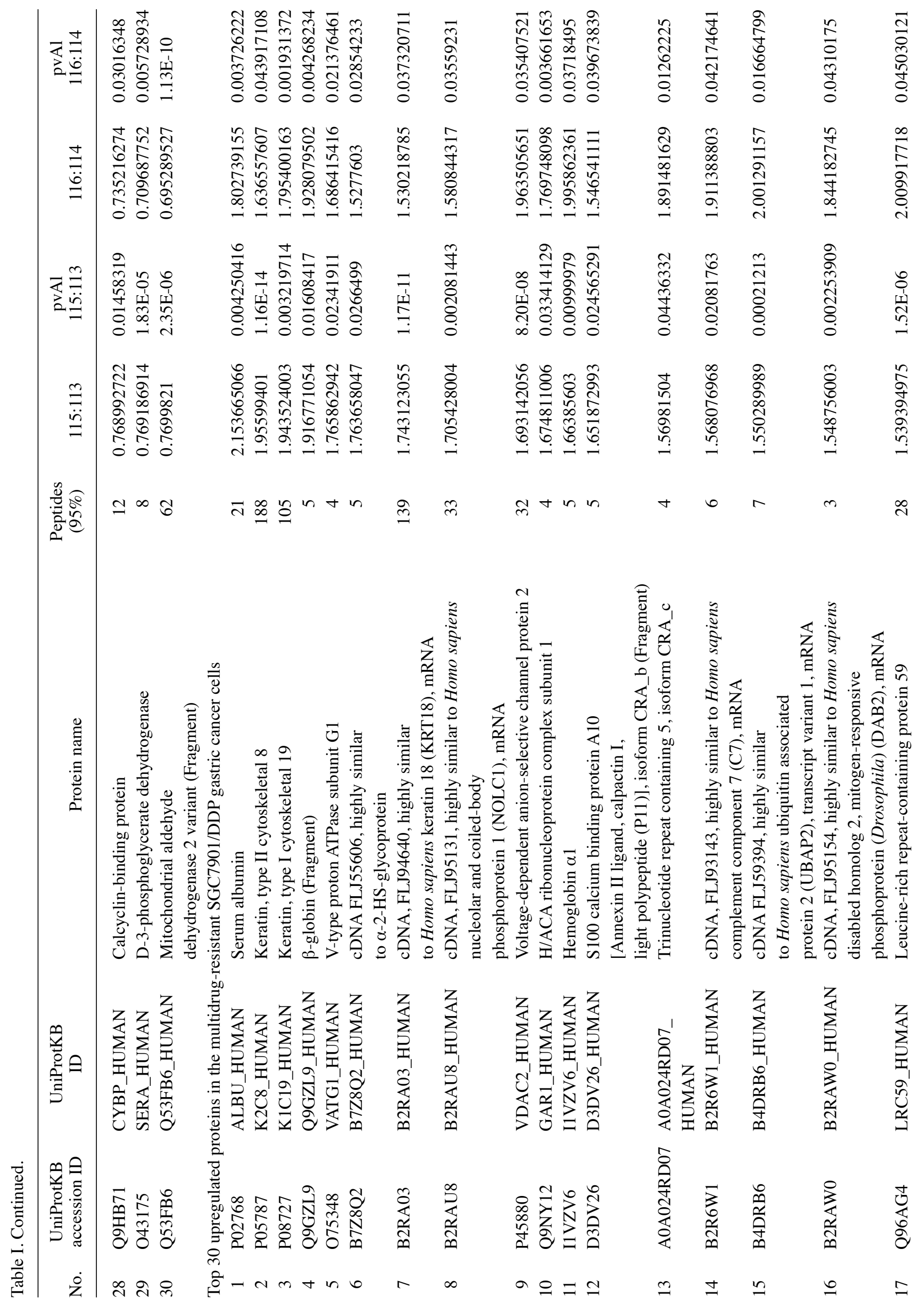




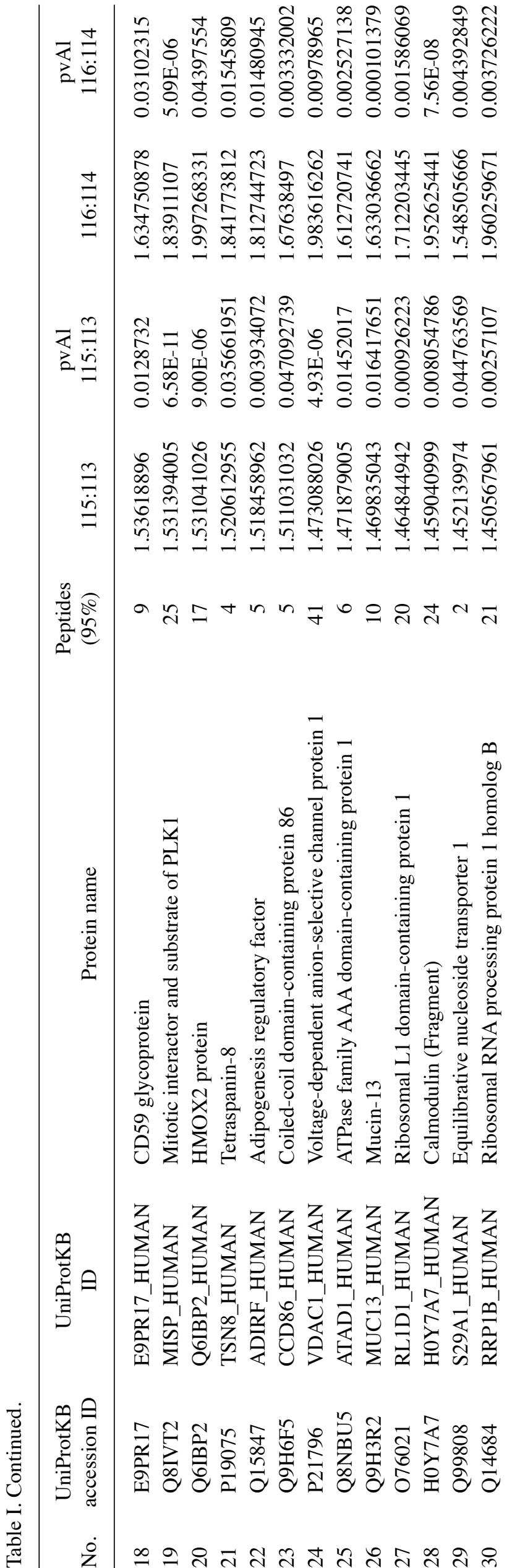

Table II. $\mathrm{IC}_{50}$ values (mg/l) for selected reagents after siRNA transfection.

\begin{tabular}{lcc}
\hline Treatment & NC & siTSPAN8 \\
\hline Cisplatin & $8.25 \pm 0.57$ & $3.89 \pm 0.27^{\mathrm{a}}$ \\
5 -Fu & $4.43 \pm 0.22$ & $2.41 \pm 0.16^{\mathrm{a}}$ \\
Adriamycin & $2.48 \pm 0.19$ & $1.54 \pm 0.10^{\mathrm{a}}$
\end{tabular}

Data are the means $\pm \mathrm{SD} .{ }^{\mathrm{a}} \mathrm{P}<0.05$ vs. the negative control $(\mathrm{NC})$ in the same treatment group. 5-Fu, 5-fluorouracil.

Table III. TSPAN8-silencing meditated reduction of $\mathrm{IC}_{50}$ could be partially reversed by $\mathrm{Wnt} / \beta$-catenin inhibitors.

\begin{tabular}{lcrcc}
\hline & & & & siTSPAN8 + siTSPAN8 + \\
Treatment & NC & siTSPAN8 & CCT036477 & XAV939 \\
\hline Cisplatin & $7.97 \pm 0.62$ & $3.77 \pm 0.41^{\mathrm{a}}$ & $5.92 \pm 0.51^{\mathrm{a}, \mathrm{b}}$ & $5.84 \pm 0.48^{\mathrm{a}, \mathrm{b}}$
\end{tabular}

Data are the means $\pm \mathrm{SD} .{ }^{\mathrm{a}} \mathrm{P}<0.05$ vs. negative control $(\mathrm{NC}) ;{ }^{\mathrm{b}} \mathrm{P}<0.05$ vs. siTSPAN8 group. TSPAN8, tetraspanin-8.

\section{Discussion}

Gastric cancer is one of the most common malignant tumors worldwide (31), and is the leading cause of morbidity and mortality among malignant tumors in East Asia (32). Unfortunately, the majority of patients are diagnosed at the advanced stages of the disease, when chemotherapy is regarded as an important component of multimodal treatment (33). Platinum- or fluorouracil-based chemotherapy is established as the first-line treatment for patients with advanced gastric cancer (34). Cisplatin and other platinum-based cancer drugs destroy tumor cells by binding to DNA strands and interfering with DNA replication $(33,34)$. While cisplatin is often effective when first administered, clinical drug resistance to cisplatin-based chemotherapy is considered a major impediment in the treatment of patients with gastric cancer $(3,34)$. Drug resistance in cancer patients includes the development of intrinsic or acquired drug resistance against chemotherapeutic agents (35). The resistance phenotype is associated with cancer cells gaining a cross-resistance to a large range of drugs that are structurally and functionally different, referred to as MDR (36). The mechanisms of MDR in cancer remain understood on only a limited basis. A wide range of mechanisms contribute to MDR, including drug efflux mediation by ATP-binding cassette $(\mathrm{ABC})$ transporter, the prevention of apoptosis, alterations in drug targets, the aberrant activation of cell signaling pathways, altered cell cycle events, cancer stem cells (CSC), epigenetic regulation, tumor microenvironment and many other causes $(8,36)$. MDR results in treatment failure or even death in patients with gastric cancer $(4,37)$ and, as such, strategies to reverse MDR have been a high priority goal in cancer research.

In the present study, we searched for proteins possibly related to drug resistance in the human gastric cancer cell 
A

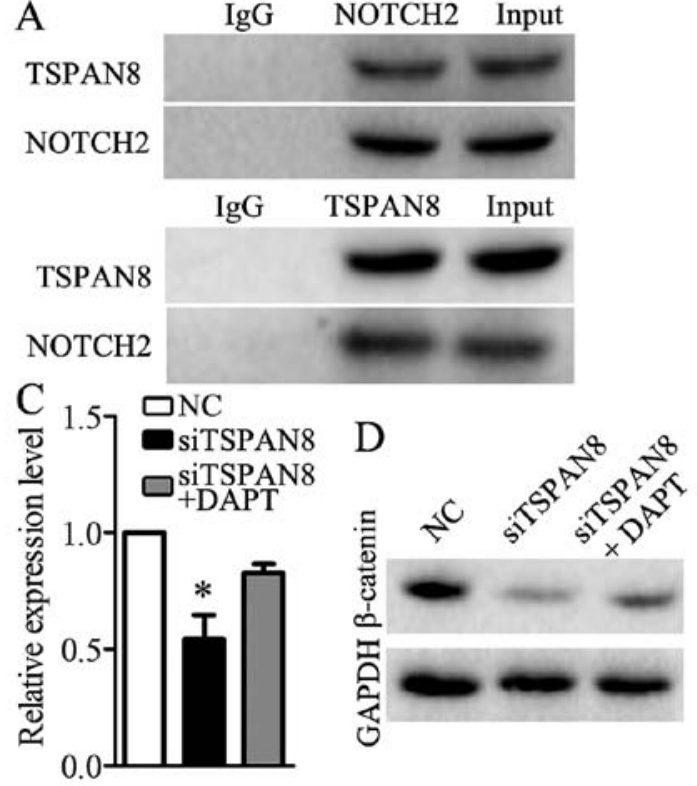

B

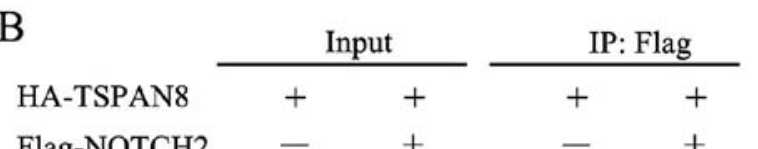

Flag-NOTCH2 $\quad-\quad+\quad-\quad+$

HA-TSPAN8

Flag-NOTCH2

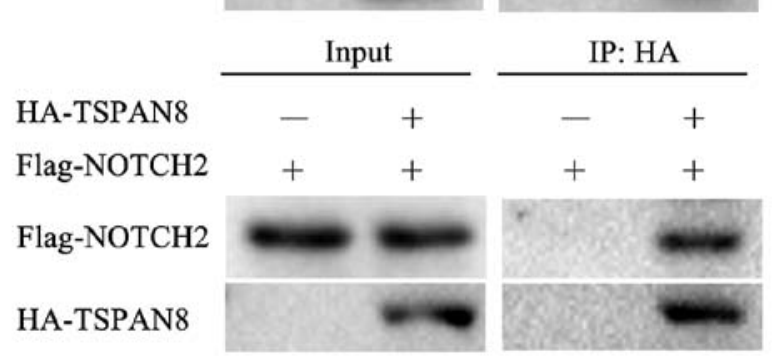

Figure 6. Tetraspanin-8 (TSPAN8) mediates $\beta$-catenin expression by binding to NOTCH2. (A) Co-immunoprecipitation of endogenous TSPAN8 and NOTCH2 . Non-denatured cell extracts prepared from SGC7901/DDP cells were immunoprecipitated with anti-TSPAN8, anti-NOTCH2 or control IgG antibody. The immune complexes and the input (10\% of cells extracts used in the immunoprecipitation step) were analyzed by immunoblotting with antibody specific to TSPAN8 (the first panel) or NOTCH2 (the fourth panel). The same membrane was stripped and reprobed to detect NOTCH2 (the second panel) or TSPAN8 (the third panel). (B) Immunoblot analysis of extracts after immunoprecipitation from SGC7901/DDP cells transfected with HA-TSPAN8 and Flag-NOTCH2. When NOTCH2 was blocked by DAPT, the TSPAN8-mediated downregulation of $\beta$-catenin was partially reversed at both (C) the mRNA level and (D) protein level. Data are the means $\pm \mathrm{SD} ;{ }^{*} \mathrm{P}<0.05$ vs. negative control (NC).

lines, SGC7901 and SCG7901/DDP, using iTRAQ-based quantitative proteomics. In total, 64 proteins were found to be increased, while 48 proteins were found to be decreased, in the SGC7901/DDP cancer cells, compared with the drug-sensitive SGC7901 cells. VDAC2, TPD53, MUC13 and HSP90 (38-41) have been previously reported to be closely associated with MDR. Thus, these proteins and another 4 of the mostly dysregulated proteins were selected for validation. Western blot analysis revealed that the expression levels of TSPAN8, VDAC2, TPD54, MUC13, HSP90, YA61, EPB41 and CRABP2 were validated at the same levels as those obtained from the results of the quantitative proteomic analysis, confirming that the iTRAQ-based quantitative proteomics is an efficient and powerful method for the analysis of MDR-related proteins. TSPAN8 expression was found to be significantly increased in the SGC7901/DDP cells, the drug-resistant gastric cancer cell line. The overexpression of TSPAN8 has been reported in many types of cancer, including hepatocellular carcinoma, pancreatic cancer, colon carcinoma and gastric cancer $(14-15,20,42)$. TSPAN8 has been implicated as increasing the proliferation, migration and invasion of many types of cancer cells, including gastric cancer cells (20). However, the role of TSPAN8 in the MDR of gastric cancer cells remains unknown. In this study, the iTRAQ-based quantitative proteomics data indicated that TSPAN8 contributed to MDR in the SGC7901/DDP cells. To confirm this, we silenced TSPAN8 in the SGC7901/DDP cells via RNA interference. The $\mathrm{IC}_{50}$ results revealed that the silencing of TSPAN8 increased the response of the gastric cancer cells to the anticancer drugs. The silencing of TSPAN8 also increased cell apoptosis. These results indicated that TSPAN8 facilitates the MDR of SGC7901/DDP cells by suppressing apoptosis.
The aberrant activation of the Wnt/ $\beta$-catenin pathway leads to cancer cell invasion, migration and $\operatorname{MDR}(27,43)$. Thus, in this study, Wnt/ $\beta$-catenin activity was monitored in the TSPAN8silenced cells. The results revealed that silencing TSPAN8 significantly decreased Wnt activity and $\beta$-catenin expression in the SGC7901/DDP cells. We also found that the $\mathrm{IC}_{50}$ of the SGC7901/DDP cells treated with cisplatin was decreased when TSPAN8 was silenced; however, this effect of TSPAN8 silencing was partially reversed when $\mathrm{Wnt} / \beta$-catenin pathway inhibitors were used. All these data indicated that TSPAN8 enhanced the resistance of SGC7901/DDP cells to chemotherapy through the activation of the $\mathrm{Wnt} / \beta$-catenin pathway and by increasing $\beta$-catenin expression and accumulation in the nucleus. When the Wnt/ $\beta$-catenin pathway is aberrantly activated, the transcription of downstream genes mediated by Wnt signaling increases. A number of Wnt targeting genes, such as LEF1 and c-MYC, induce drug resistance in cancer cells $(44,45)$. This explains how TSPAN8 increases the MDR of SGC7901/DDP cells by mediating the Wnt/ $\beta$-catenin pathway.

To further explore the mechanisms of action of TSPAN8 as regards MDR, we searched the biological database. It was predicted that TSPAN8 may interact with NOTCH2 (46), which has been reported to participate in Wnt/ $\beta$-catenin-based MDR in osteosarcoma (31). We hypothesized that TSPAN8 mediated the activation of the $\mathrm{Wnt} / \beta$-catenin pathway by binding to NOTCH2 in SGC7901/DDP cells. Co-immunoprecipitation revealed that TSPAN8 bound to NOTCH2. The impairment of $\beta$-catenin expression was partially compensated when DAPT, a NOTCH2 inhibitor, was used in TSPAN8-silenced SGC7901/DDP cells. These data indicated that TSPAN8 mediated $\mathrm{Wnt} / \beta$-catenin pathway activation by binding to $\mathrm{NOTCH} 2$. However, further studies are warranted in order to 
elucidate the mechanisms through which TSPAN8 interacts with NOTCH2 in MDR. Taken together, our study indicates that the inhibition of TSPAN8 sensitizes gastric cancer cells to chemotherapeutic drugs. However, to obtain a more complete picture of the molecular mechanisms involved in the regulation of the MDR of SGC7901/DDP by TSPAN8, further studies are required in the future.

In conclusion, the present study demonstrates that TSPAN8 impairs the sensitivity of SGC7901/DDP gastric cancer cells to chemotherapeutic agents by mediating $\mathrm{Wnt} / \beta$-catenin activity. TSPAN8 also mediates $\beta$-catenin expression and accumulation by binding to NOTCH2. This study provides novel insight for drug designs that overcome cisplatin resistance in gastric cancer cells.

\section{Acknowledgements}

This study was supported by the Foundation for Young Scientists of Guizhou Provincial People's Hospital [grant no. GZSYQN (2016) 19] and the Foundation of Health and Family Planning Commission of Guizhou Province (grant no. GZWJKT2015-1-022).

\section{References}

1. Kamangar F, Dores GM and Anderson WF: Patterns of cancer incidence, mortality, and prevalence across five continents: Defining priorities to reduce cancer disparities in different geographic regions of the world. J Clin Oncol 24: 2137-2150, 2006.

2. Siegel RL, Miller KD and Jemal A: Cancer statistics, 2016. CA Cancer J Clin 66: 7-30, 2016.

3. Ajani JA, Ota DM, Jessup JM, Ames FC, McBride C, Boddie A, Levin B, Jackson DE, Roh M and Hohn D: Resectable gastric carcinoma. An evaluation of preoperative and postoperative chemotherapy. Cancer 68: 1501-1506, 1991

4. Zhang D and Fan D: Multidrug resistance in gastric cancer: Recent research advances and ongoing therapeutic challenges. Expert Rev Anticancer Ther 7: 1369-1378, 2007

5. Kartal-Yandim M, Adan-Gokbulut A and Baran Y: Molecular mechanisms of drug resistance and its reversal in cancer. Crit Rev Biotechnol 36: 716-726, 2016.

6. Borst P: Genetic mechanisms of drug resistance. A review. Acta Oncol 30: 87-105, 1991.

7. Cui J, Jiang W, Wang S, Wang L and Xie K: Role of Wnt/ $\beta$-catenin signaling in drug resistance of pancreatic cancer. Curr Pharm Des : 2464-2471, 2012.

8. Abdi J, Chen G and Chang H: Drug resistance in multiple myeloma: Latest findings and new concepts on molecular mechanisms. Oncotarget 4: 2186-2207, 2013.

9. Yang Z, Guo L, Liu D, Sun L, Chen H, Deng Q, Liu Y, Yu M, Ma Y, Guo N, et al: Acquisition of resistance to trastuzumab in gastric cancer cells is associated with activation of IL-6/STAT3/ Jagged-1/Notch positive feedback loop. Oncotarget 6: 5072-5087, 2015.

10. McAuliffe SM, Morgan SL, Wyant GA, Tran LT, Muto KW, Chen YS, Chin KT, Partridge JC, Poole BB, Cheng KH, et al: Targeting Notch, a key pathway for ovarian cancer stem cells, sensitizes tumors to platinum therapy. Proc Natl Acad Sci USA 109: E2939-E2948, 2012.

11. Ren T, Lin S, Wang Z and Shang A: Differential proteomics analysis of low- and high-grade of astrocytoma using iTRAQ quantification. Onco Targets Ther 9: 5883-5895, 2016.

12. Berthier-Vergnes $\mathrm{O}$, El Kharbili $\mathrm{M}$, de la Fouchardière $\mathrm{A}$, Pointecouteau T, Verrando P, Wierinckx A, Lachuer J, Le Naour F and Lamartine J: Gene expression profiles of human melanoma cells with different invasive potential reveal TSPAN8 as a novel mediator of invasion. Br J Cancer 104: 155-165, 2011.

13. Yue S, Mu W, Erb U and Zöller M: The tetraspanins CD151 and Tspan 8 are essential exosome components for the crosstalk between cancer initiating cells and their surrounding. Oncotarget 6: 2366-2384, 2015.
14. Kanetaka K, Sakamoto M, Yamamoto Y, Yamasaki S, Lanza F, Kanematsu T and Hirohashi S: Overexpression of tetraspanin CO-029 in hepatocellular carcinoma. J Hepatol 35: 637-642, 2001.

15. Gesierich S, Paret C, Hildebrand D, Weitz J, Zgraggen K, Schmitz-Winnenthal FH, Horejsi V, Yoshie O, Herlyn D, Ashman LK, et al: Colocalization of the tetraspanins, CO-029 and CD151, with integrins in human pancreatic adenocarcinoma: Impact on cell motility. Clin Cancer Res 11: 2840-2852, 2005.

16. Hemler ME: Tetraspanin functions and associated microdomains. Nat Rev Mol Cell Biol 6: 801-811, 2005.

17. Yáñez-Mó M, Barreiro O, Gordon-Alonso M, Sala-Valdés M and Sánchez-Madrid F: Tetraspanin-enriched microdomains: A functional unit in cell plasma membranes. Trends Cell Biol 19: 434-446, 2009

18. Zöller M: Tetraspanins: Push and pull in suppressing and promoting metastasis. Nat Rev Cancer 9: 40-55, 2009.

19. Nazarenko I, Rana S, Baumann A, McAlear J, Hellwig A, Trendelenburg M, Lochnit G, Preissner KT and Zöller M: Cell surface tetraspanin Tspan8 contributes to molecular pathways of exosome-induced endothelial cell activation. Cancer Res 70: 1668-1678, 2010.

20. Wei L, Li Y and Suo Z: TSPAN8 promotes gastric cancer growth and metastasis via ERK MAPK pathway. Int J Clin Exp Med 8: 8599-8607, 2015

21. Mao ZL, He SB, Sheng WH, Dong XQ and Yang JC: Adenovirusmediated ING4 expression reduces multidrug resistance of human gastric carcinoma cells in vitro and in vivo. Oncol Rep 30: 2187-2194, 2013

22. Soothill JS, Ward R and Girling AJ: The IC50: An exactly defined measure of antibiotic sensitivity. J Antimicrob Chemother 29: 137-139, 1992.

23. Livak KJ and Schmittgen TD: Analysis of relative gene expression data using real-time quantitative PCR and the $2(-\Delta \Delta \mathrm{C}(\mathrm{T}))$ method. Methods 25: 402-408, 2001.

24. Mi H, Muruganujan A and Thomas PD: PANTHER in 2013: Modeling the evolution of gene function, and other gene attributes, in the context of phylogenetic trees. Nucleic Acids Res 41: D377-D386, 2013

25. Szklarczyk D, Franceschini A, Wyder S, Forslund K, Heller D, Huerta-Cepas J, Simonovic M, Roth A, Santos A, Tsafou KP, et al: STRING v10: Protein-protein interaction networks, integrated over the tree of life. Nucleic Acids Res 43: D447-D452, 2015.

26. Gan CS, Chong PK, Pham TK and Wright PC: Technical, experimental, and biological variations in isobaric tags for relative and absolute quantitation (iTRAQ). J Proteome Res 6: 821-827, 2007.

27. Gong J, Shen S, Yang Y, Qin S, Huang L, Zhang H, Chen L, Chen Y, Li S, She S, et al: Inhibition of FASN suppresses migration, invasion and growth in hepatoma carcinoma cells by deregulating the HIF-1 $\alpha /$ IGFBP1 pathway. Int J Oncol 50: 883-892, 2017.

28. Mitra A, Mishra L and Li S: EMT, CTCs and CSCs in tumor relapse and drug-resistance. Oncotarget 6: 10697-10711, 2015.

29. Clements WM, Wang J, Sarnaik A, Kim OJ, MacDonald J, Fenoglio-Preiser C, Groden J and Lowy AM: $\beta$-Catenin mutation is a frequent cause of Wnt pathway activation in gastric cancer. Cancer Res 62: 3503-3506, 2002.

30. Ma Y, Ren Y, Han EQ, Li H, Chen D, Jacobs JJ, Gitelis S, O'Keefe RJ, Konttinen YT, Yin G, et al: Inhibition of the Wnt- $\beta$-catenin and Notch signaling pathways sensitizes osteosarcoma cells to chemotherapy. Biochem Biophys Res Commun 431: 274-279, 2013.

31. Hartgrink HH, Jansen EPM, van Grieken NCT and van de Velde CJ: Gastric cancer. Lancet 374: 477-490, 2009.

32. Loomis D, Huang W and Chen G: The International Agency for Research on Cancer (IARC) evaluation of the carcinogenicity of outdoor air pollution: Focus on China. Chin J Cancer 33: 189-196, 2014.

33. Wagner AD, Grothe W, Haerting J, Kleber G, Grothey A and Fleig WE: Chemotherapy in advanced gastric cancer: A systematic review and meta-analysis based on aggregate data. J Clin Oncol 24: 2903-2909, 2006

34. Kim DY, Kim JH, Lee SH, Kim TY, Heo DS, Bang YJ and Kim NK: Phase II study of oxaliplatin, 5-fluorouracil and leucovorin in previously platinum-treated patients with advanced gastric cancer. Ann Oncol 14: 383-387, 2003.

35. Shen DW, Pouliot LM, Hall MD and Gottesman MM: Cisplatin resistance: A cellular self-defense mechanism resulting from multiple epigenetic and genetic changes. Pharmacol Rev 64: 706-721, 2012. 
36. Krishna $\mathrm{R}$ and Mayer LD: Multidrug resistance (MDR) in cancer. Mechanisms, reversal using modulators of MDR and the role of MDR modulators in influencing the pharmacokinetics of anticancer drugs. Eur J Pharm Sci 11: 265-283, 2000.

37. Endo K, Maehara Y, Kusumoto T, Ichiyoshi Y, Kuwano M and Sugimachi K: Expression of multidrug-resistance-associated protein (MRP) and chemosensitivity in human gastric cancer. Int J Cancer 68: 372-377, 1996

38. Yang YX, Xiao ZQ, Chen ZC, Zhang GY, Yi H, Zhang PF, $\mathrm{Li}$ JL and Zhu G: Proteome analysis of multidrug resistance in vincristine-resistant human gastric cancer cell line SGC7901/ VCR. Proteomics 6: 2009-2021, 2006.

39. McCubrey JA, Lertpiriyapong K, Fitzgerald TL, Martelli AM, Cocco L, Rakus D, Gizak A, Libra M, Cervello M, Montalto G, et al: Roles of TP53 in determining therapeutic sensitivity, growth, cellular senescence, invasion and metastasis. Adv Biol Regul 63: 32-48, 2017.

40. Sheng Y, Ng CP, Lourie R, Shah ET, He Y, Wong KY, Seim I, Oancea I, Morais C, Jeffery PL, et al: MUC13 overexpression in renal cell carcinoma plays a central role in tumor progression and drug resistance. Int J Cancer 140: 2351-2363, 2017.

41. Wang J, Zhang Y, Liu T, Guo CH and Wan YF: Reversal effect of inhibition of HSP90 activity on adriamycin resistance of human hepatocellular carcinoma HepG2/ADR cells. Tumor 36: 414-423, 2016.
42. Ren YP, Song C and Meng QK: Expression of Tspan8 in colon cancer cell lines and effects of siRNA-mediated Tspan8 gene silencing on the cell function of SW620. Zhongguo Yike Daxue Xuebao 44: 34-37, 2015 (In Chinese).

43. Castillo V, Valenzuela R, Huidobro C, Contreras HR and Castellon EA: Functional characteristics of cancer stem cells and their role in drug resistance of prostate cancer. Int J Oncol 45: 985-994, 2014

44. Singh A and Settleman J: EMT, cancer stem cells and drug resistance: An emerging axis of evil in the war on cancer. Oncogene 29: 4741-4751, 2010.

45. Niimi S, Nakagawa K, Yokota J, Tsunokawa Y, Nishio K, Terashima Y, Shibuya M, Terada M and Saijo N: Resistance to anticancer drugs in NIH3T3 cells transfected with c-myc and/or c-H-ras genes. Br J Cancer 63: 237-241, 1991.

46. Hayward P, Brennan K, Sanders P, Balayo T, DasGupta R, Perrimon N and Martinez Arias A: Notch modulates Wnt signalling by associating with Armadillo/ $\beta$-catenin and regulating its transcriptional activity. Development 132: 1819-1830, 2005. 